\title{
Novel approach of vaccination against Brucella abortus 544 based on a combination of fusion proteins, human serum albumin and brucella abortus Lipopolysaccharides.
}

\begin{abstract}
Lipopolysaccharide (LPS) of Brucella abortus is an essential component for developing the subunit vaccine against brucellosis. B. abortus LPS was extracted by n-butanol, purified by ultracentrifugation and detoxified by alkaline treatment. Pyrogenicity and toxicity of $\mathrm{B}$. abortus LPS and detoxified-LPS (D-LPS) were analyzed and compared with LPS of E. coli. Different groups of mice were immunized intraperitoneally with purified B. abortus LPS, DLPS, a combination of LPS with human serum albumin (LPS-HSA) and B. abortus S19 bacteria; besides, control mice were inoculated with sterile saline. Two doses of vaccine were given 4 weeks apart. Mice were challenged intraperitoneally with virulent B. abortus 544 strain 4 weeks after the second dose of vaccine. Sera and spleens of mice were harvested 4 weeks after challenge. LPS-B. abortus was 10,000-fold less potent in LAL test and 100-fold less potent in eliciting fever in rabbits than in E. coli LPS. And D-LPS was very less potent in LAL test and eliciting fever in rabbits ordinary LPS. The antibody titer of anti-LPS immunoglobulin G (IgG) was higher than D-LPS. However, mice immunized with either LPS, D-LPS or LPS-HSA vaccines showed a significant protection against infection of the spleen $(p<0.01)$. There was no significant difference between mice immunized with LPS and D-LPS in terms of protection $(\mathrm{p}<0.99)$. Therefore, it was concluded that D-LPS and LPS-HSA for B. abortus can be used as safer and more potent vaccines than ordinary LPS-B. abortus vaccine.
\end{abstract}

Keyword: Novel approach of vaccination; Brucella abortus 544; Fusion proteins; Human serum albumin; Brucella abortus lipopolysaccharides. 